Review

\title{
Novel Trends in Dental Color Match Using Different Shade Selection Methods: A Systematic Review and Meta-Analysis
}

\author{
Louis Hardan ${ }^{1}\left(\mathbb{0}, \operatorname{Rim}_{\text {Bourgi }}{ }^{1}\left(\mathbb{0}\right.\right.$, Carlos Enrique Cuevas-Suárez ${ }^{2, *} \mathbb{D}^{4}$, Monika Lukomska-Szymanska $^{3}(\mathbb{0}$, \\ Ana Josefina Monjarás-Ávila ${ }^{2}$, Maciej Zarow ${ }^{4}$, Natalia Jakubowicz ${ }^{4}$, Gilbert Jorquera ${ }^{5}{ }^{\circ}$, Tarek Ashi ${ }^{6}$, \\ Davide Mancino ${ }^{7,8}$, Naji Kharouf ${ }^{7,8, *}$ and Youssef Haikel ${ }^{7,8}$
}

check for updates

Citation: Hardan, L.; Bourgi, R.; Cuevas-Suárez, C.E.

Lukomska-Szymanska, M.

Monjarás-Ávila, A.J.; Zarow, M.;

Jakubowicz, N.; Jorquera, G.; Ashi, T.;

Mancino, D.; et al. Novel Trends in

Dental Color Match Using Different

Shade Selection Methods: A

Systematic Review and

Meta-Analysis. Materials 2022, 15

468. https://doi.org/10.3390/

ma15020468

Academic Editors: Alessandro Vichi and Bruno Chrcanovic

Received: 17 November 2021

Accepted: 6 January 2022

Published: 8 January 2022

Publisher's Note: MDPI stays neutral with regard to jurisdictional claims in published maps and institutional affiliations.

Copyright: (C) 2022 by the authors. Licensee MDPI, Basel, Switzerland. This article is an open access article distributed under the terms and conditions of the Creative Commons Attribution (CC BY) license (https:// creativecommons.org/licenses/by/ $4.0 /)$
1 Department of Restorative Dentistry, School of Dentistry, Saint-Joseph University, Beirut 1107 2180, Lebanon; louis.hardan@usj.edu.lb (L.H.); rim.bourgi@net.usj.edu.lb (R.B.)

2 Dental Materials Laboratory, Academic Area of Dentistry, Autonomous University of Hidalgo State, Circuito Ex Hacienda la Concepción S/N, San Agustín Tlaxiaca 42160, HGO, Mexico; ana_monjaras@uaeh.edu.mx

3 Department of General Dentistry, Medical University of Lodz, 251 Pomorska St., 92-213 Lodz, Poland; monika.lukomska-szymanska@umed.lodz.pl

4 “NZOZ SPS Dentist" Dental Clinic and Postgraduate Course Centre, pl. Inwalidow 7/5, 30-033 Cracow, Poland; dentist@dentist.com.pl (M.Z.); nljakubowicz@gmail.com (N.J.)

5 Department of Prosthodontics, Universidad de los Andes, Santiago 12455, Chile; gjorquera@uandes.cl

6 Private Practice, Zahnarztpraxis Ashi, Bahnhofstr 2, 09419 Thum, Germany; tarekachi@live.com

7 Department of Biomaterials and Bioengineering, INSERM UMR_S 1121, Biomaterials and Bioengineering, 67000 Strasbourg, France; endodontiefrancaise@outlook.com (D.M.); youssef.haikel@unistra.fr (Y.H.)

8 Department of Endodontics, Faculty of Dental Medicine, Strasbourg University, 67000 Strasbourg, France

* Correspondence: cecuevas@uaeh.edu.mx (C.E.C.-S.); dentistenajikharouf@gmail.com (N.K.); Tel.: +52-(771)-7-2000 (C.E.C.-S.); +33-36-6752-2841 (N.K.)

\begin{abstract}
Since color matching is considered a subjective procedure, accurate shade choice is often the most challenging stage of recreating the natural appearance of teeth. Furthermore, accurate determination of tooth color is imperative for the final outcome of dental restorations. The purpose of this research is to assess the accuracy of color match between diverse shade selection methods throughout a systematic review and meta-analysis. Two independent investigators (L.H. and R.B.) screened the literature in five electronic databases. Randomized controlled trials or in vitro papers studying the effect of using either digital shade selection or visual shade selection on the accuracy of color match were included. A total of 13 manuscripts comprised the meta-analysis. Color difference $(\Delta \mathrm{E})$ between restorations where the shade matching was performed by the conventional method was greater than those where the shade matching was performed by computerized methods $(p=0.007)$. According to the subgroup analysis, only the use of digital photographs for shade matching showed a reduction in the $(\Delta \mathrm{E})(p<0.0001)$, while the use of a spectrophotometer has no advantages over the use of visual shade guide tabs $(p=0.57)$. On the other hand, global analysis showed that incorrect shade matching was higher when the conventional method using shade guide tabs was used $(p<0.001)$, irrespective of whether a spectrophotometer or a digital camera was used $(p<0.001)$. This study concluded that the use of digital photography and spectrophotometric measurements led to fewer color differences and less incorrect shade matching than conventional methods using color shade tabs.
\end{abstract}

Keywords: color; dental shade; digital dentistry; shade selection; smartphone

\section{Introduction}

The fabrication of indirect restorations necessitates worthy interaction between clinicians and laboratory technicians. Furthermore, shade choice is the often the most challenging stage in the recreation of the natural appearance of teeth [1]. According to previous studies, differences among practitioners concerning shade matching for the same teeth occur over days [2,3]. In fact, researchers have considered color matching as a subjective procedure, reliant on several influences, such as light source, object, and observer [4]. While 
there is no process considered as the gold standard, different methods to assess color in dentistry exist, including visual and instrumental [5].

Color communication using shade guides is the most shared technique. However, this method is considered subjective, since it is induced by age, sex, experience of the observer, eye fatigue, and ambient light [6]. In this sense, instrumental methods have gained popularity; however, they are expensive and not always available to the dentist [2,7]. It is important to note that instrumental methods include spectrophotometers, scanners, cross-polarizing filters, digital cameras, and smartphones [2,8]. These devices consist of a detector, signal conditioner, and software that process the signal to make the data usable in a clinic or laboratory [9]. Spectrophotometric color measurements are capable of reliably quantifying the color of both extracted teeth and dental materials [10].

Digital photographs are a common interaction tool in dental workplaces [11]. Digital photographs are able to capture clear images of the tooth and have been progressively used to document the whole clinical procedure [2,12]. Predictably, digital photographs of natural teeth can show noteworthy color modifications when the optimal conditions of light illumination are not used [12].

On the other hand, there has been an increased awareness for aesthetically pleasing restorations among patients. Hence, it is a practitioner's responsibility to provide restorations which can adequately mimic the surrounding natural dentition [13]. As scientific evidence comparing differences in visual shade matching using different devices is scarce, this topic remains controversial among researchers. Therefore, the purpose of the present study is to systematically review the literature to compare the accuracy of color matching between different shade selection methods. The null hypothesis tested was that all different shade selection methods would have the same accuracy.

\section{Materials and Methods}

This systematic review and meta-analysis were implemented in agreement with the PRISMA instructions [14]. The registration protocol was carried out in Prospero with the registration number CRD42021288077. The resulting PICOS framework performed is described in Table 1. The research question was: "Is the use of spectroscopic or camera method in dentistry accurate for color selection of the tooth?"

Table 1. PICOS framework used.

\begin{tabular}{cc}
\hline Population & Color of the Tooth \\
\hline Intervention & Spectroscopic or camera method for color determination \\
\hline Control & Clinical perception using a shade tab guide \\
\hline Outcome & Shade match \\
\hline Study design & Randomized clinical trials and in vitro studies \\
\hline
\end{tabular}

\subsection{Literature Search}

A literature search was conducted by two independent authors (L.H. and R.B.) up to July 15, 2021. Different electronic databases were selected: PubMed MedLine, Scopus, ISI Web of Science, Cochrane library, and EMBASE. These databases were used for identifying articles that could fit the specific criteria. The keywords, as well as the search strategy performed in PubMed, are summarized in Table 2. The search strategies for Scopus, ISI Web of Science, Cochrane library, and EMBASE databases are presented as Supplementary Materials (Tables S1-S4). The investigators manually checked the list of references of each included paper for the discovery of additional manuscripts. After the search, the articles were introduced into Mendeley Desktop 1.17.11 software (Glyph \& Cog, LLC, London, UK) to eliminate duplicates. 
Table 2. Search strategy used in PubMed.

\begin{tabular}{cc}
\hline Color OR Color measurement OR Colorimeters OR Spectrophotometers \\
OR spectrophotometer OR CIE L*a*b* OR Tooth Color OR Color Shade \\
OR dental color OR spectrophotometry OR colorimetry OR color \\
perception OR color matching OR color accuracy OR spectroradiometry \\
OR color*
\end{tabular}

\subsection{Study Selection}

Two researchers (L.H. and R.B.) independently assessed the abstracts and titles of all the articles. Studies for full-text review were chosen based on the following eligibility criteria: (1) randomized controlled trial or in vitro papers studying the effect of using either digital shade selection or visual shade selection on the accuracy of color selection; (2) publishing in the English language; (3) assessing the accuracy of color measurement of the tooth and/or restorative material; (4) including a numerical value for the difference in color of the subject selected. Case series, reviews, case reports, and pilot studies were excluded. Full versions of any potentially suitable manuscripts were analyzed. Manuscripts that met the inclusion conditions or had inadequate data in the abstract and title to provide a clear judgment were considered for full evaluation. Full-text papers were evaluated by independent reviewers. Any variations in the decision-making process in regard to the suitability of the accepted articles was resolved and decided upon through the consensus of a third author (C.E.C.-S.). Only manuscripts that satisfied all of the eligibility criteria recorded were integrated for assessment.

\subsection{Data Extraction}

The information of concern from the papers included was organized using a harmonized form in a Microsoft Office Excel 2019 spreadsheet (Microsoft Corporation, Redmond, WA, USA). These records comprised demographic data (author and year of publication), method used for shade match, materials used, type of room light, the main outcome, and main results. The corresponding authors of the included articles were communicated twice via e-mail to regain any missing data. If the investigators did not answer within 10 days of the first communication, the omitted data was not incorporated.

\subsection{Quality Assessment}

The methodological quality of each involved manuscript was independently assessed by two investigators (R.B. and L.H.), considering the parameters of the Cochrane Collaboration's tool for evaluating risk of bias in randomized trials [15]. For the in vitro articles, the risk of bias in each manuscript was assessed according to the description of the subsequent parameters: specimen randomization, single operator, operator blinded, control group, standardized specimens, and sample size calculation [15]. If the reviewers stated the parameter, the study expected a "YES" of that precise parameter. In case of missing information, the parameter received a "NO." The risk of bias was categorized conferring to the sum of "YES" answers received: 1 to 2 designated a high bias, 3 to 4 medium, and 5 to 6 showed a low risk of bias. For the clinical studies, the risk of bias in each paper was considered according to the description of the subsequent parameters: allocation concealment, incomplete outcome data, sequence generation, blinding of the outcome assessors, selective outcome reporting, and other potential sources of bias. All these areas were assessed at the 
analysis level. Through risk of bias evaluation, any discrepancies between the researchers were solved through argument by accessing a third referee (C.E.C.-S.).

\subsection{Statistical Analysis}

Meta-analyses were completed using the Review Manager Software version 5.3.5 (The Nordic Cochrane Centre, The Cochrane Collaboration, Copenhagen, Denmark). For the quantitative data, a random-effect model was conducted for evaluates, and pooledeffect estimates were acquired by comparing the standardized mean difference of the $(\Delta \mathrm{E})$ between the classical shade guide and the computerized methods. Subgroup analyses were accomplished using a spectrophotometer or a digital camera to assess the shade selection. For the qualitative data, global analysis was conducted using a fixed-effects model, and pooled-effect estimates were acquired by comparing the risk difference of incorrect shade matching of the computerized methods (use of spectrophotometer or digital camera), or the conventional visual shade guide. A $p$-value of $<0.05$ was considered statistically significant. Statistical heterogeneity of the treatment effect among studies was evaluated using the Cochran $\mathrm{Q}$ test and the inconsistency $\mathrm{I}^{2}$ test.

\section{Results}

A total of 4136 papers were obtained from the databases (Figure 1). After removing the duplicates, the total quantity of literature found was 3299 publications for the primary examination. Then, 3268 manuscripts were excluded after revising the titles and summaries, leaving a total of 31 articles to be assessed for full-text review. Of these, 18 studies were excluded [16-33]. Exclusion reasons are assumed in the PRISMA flow diagram of the research, which resulted in a total of 13 manuscripts in the meta-analysis [34-46]. These manuscripts included seven in vitro studies and six clinical trials.

\section{PRISMA FLOW DIAGRAM}

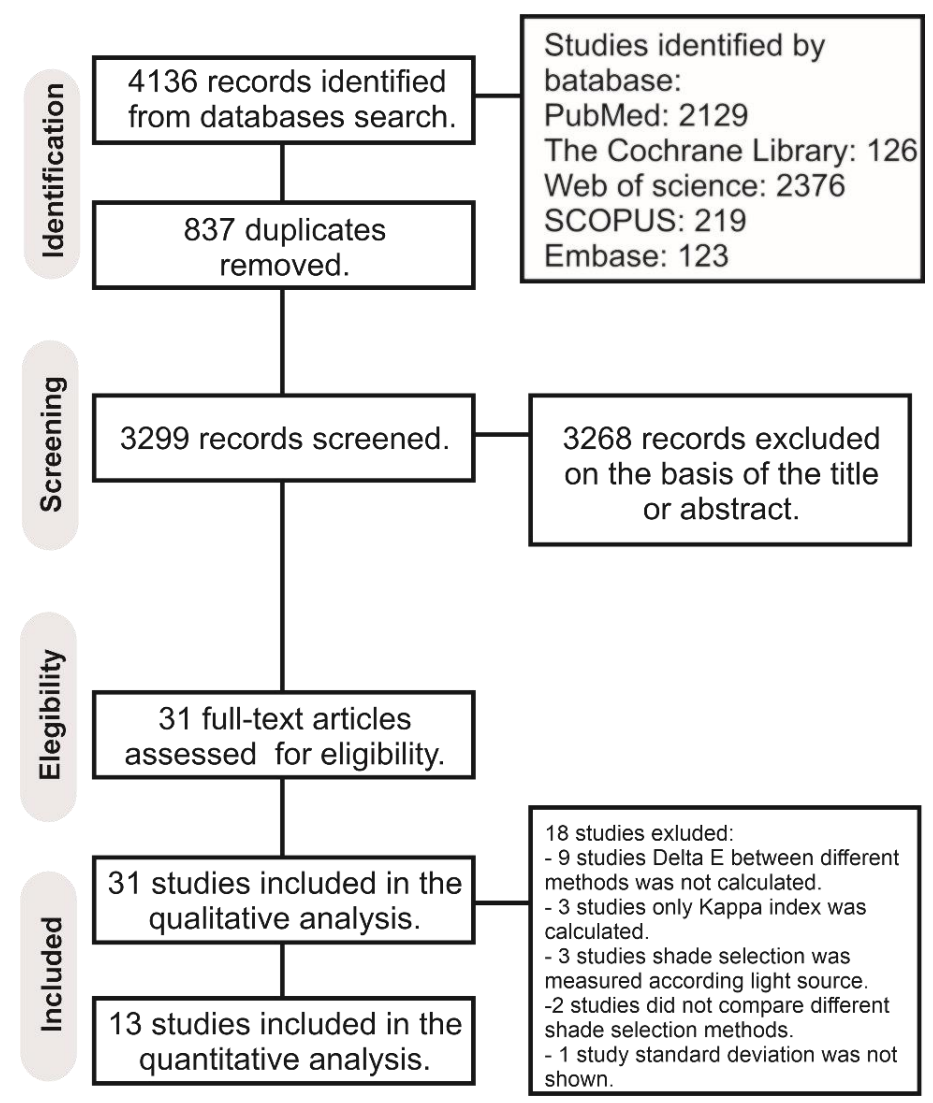

Figure 1. Prisma flow diagram of the research. 
The characteristics of the manuscripts incorporated in this systematic review were recapitulated in Table 3.

Table 3. Demographic and study design data of the incorporated manuscripts.

\begin{tabular}{|c|c|c|c|c|c|c|}
\hline Study & & $\begin{array}{l}\text { Method Used for } \\
\text { Shade Match }\end{array}$ & Materials Used & Type of Room Light & $\begin{array}{l}\text { Main } \\
\text { Outcome }\end{array}$ & Main Results \\
\hline $\begin{array}{l}\text { Jorquera, } \\
2021\end{array}$ & $\begin{array}{l}1 . \\
2 .\end{array}$ & $\begin{array}{l}\text { Shade guide Table } \\
\text { Digital camera and } \\
\text { cross-polarizing filter. } \\
\text { Smartphone with a } \\
\text { light-correcting } \\
\text { device. }\end{array}$ & Human tooth & $\begin{array}{c}\text { The room had ambient } \\
\text { light between } 5500 \mathrm{~K} \\
\text { and } 6500 \mathrm{~K} \text {. } \\
\text { The clothing of the } \\
\text { volunteers was covered } \\
\text { with a neutral } \\
\text { color cloth. }\end{array}$ & $\Delta \mathrm{E}$ & $\begin{array}{c}\text { Digital shade choice using both } \\
\text { a digital camera and a } \\
\text { smartphone displayed a } \\
\text { threshold within the adequate } \\
\text { values }(\Delta \mathrm{E}<3.7) \text {. } \\
\text { Visual shade choice displayed } \\
\text { an average } \Delta \mathrm{E} \text { above the level } \\
\text { for acceptable values }(\Delta \mathrm{E}>3.7) \text {. }\end{array}$ \\
\hline Mahn, 2021 & $\begin{array}{l}1 . \\
2 . \\
3 .\end{array}$ & $\begin{array}{l}\text { Shade guide tab. } \\
\text { Digital camera with a } \\
\text { cross-polarized filter. } \\
\text { Spectrophotometer }\end{array}$ & Human tooth & $\begin{array}{c}\text { The room had ambient } \\
\text { light between } 5500 \mathrm{~K} \\
\text { and } 6500 \mathrm{~K} \text {. } \\
\text { The clothing of the } \\
\text { volunteers was covered } \\
\text { with a neutral } \\
\text { color cloth. }\end{array}$ & $\Delta \mathrm{E}$ & $\begin{array}{c}\text { No statistically significant } \\
\text { differences within digital } \\
\text { images and spectrophotometer. } \\
\text { The visual shade process led to } \\
\text { enormous variances compared } \\
\text { to the other approaches } \\
\text { beneath study. }\end{array}$ \\
\hline $\begin{array}{l}\text { Sampaio, } \\
2019\end{array}$ & 1. & $\begin{array}{l}\text { Digital camera with } \\
\text { ring flash or a } \\
\text { dual-point rigid } \\
\text { flash bracket. } \\
\text { Cross-polarizing } \\
\text { filter attached to a } \\
\text { close-up flash. } \\
\text { iPhone } 7 \text {. }\end{array}$ & Human tooth & $\begin{array}{l}\text { For comparison, the } \\
\text { measurements were } \\
\text { made in the same spots. }\end{array}$ & $\Delta \mathrm{E}$ & $\begin{array}{l}\text { Using a cross-polarizing filter } \\
\text { consequences in more } \\
\text { color-standardized } \\
\text { photographs; however, using an } \\
\text { iPhone } 7 \text { and a ring flash } \\
\text { system resulted in fewer } \\
\text { standardized pictures. }\end{array}$ \\
\hline $\begin{array}{l}\text { Zekonis, } \\
2002\end{array}$ & $\begin{array}{l}1 . \\
2 . \\
3 .\end{array}$ & $\begin{array}{l}\text { Colorimeter } \\
\text { Shade guide } \\
\text { Color slide } \\
\text { photography }\end{array}$ & Human tooth & Non-specified & $\Delta \mathrm{E}$ & $\begin{array}{l}\text { At-home }(10 \% \text { carbamide } \\
\text { peroxide) treatment sides were } \\
\text { meaningfully distinctive from } \\
\text { the in-office ( } 35 \% \text { hydrogen } \\
\text { peroxide) treatment sides } \\
\text { through all active treatment } \\
\text { stages and during follow-up } \\
\text { visits conferring to all three } \\
\text { color assessment approaches. }\end{array}$ \\
\hline Matis, 2002 & $\begin{array}{l}1 . \\
2 . \\
3 .\end{array}$ & $\begin{array}{l}\text { Shade guide. } \\
\text { Digital photographs } \\
\text { Color-measuring } \\
\text { device }\end{array}$ & Human tooth & Non-specified & $\Delta \mathrm{E}$ & $\begin{array}{l}\text { Shade guide and slide } \\
\text { photography data displayed no } \\
\text { meaningful variances between } \\
\text { teeth lightened with agent with } \\
\text { or without reservoirs. }\end{array}$ \\
\hline Jarad, 2003 & $\begin{array}{l}1 . \\
2 .\end{array}$ & $\begin{array}{l}\text { Shade guides. } \\
\text { Digital camera. }\end{array}$ & $\begin{array}{l}\text { Porcelain shade } \\
\text { tabs }\end{array}$ & $\begin{array}{l}\text { All background } \\
\text { lighting in the room } \\
\text { was maintained at a } \\
\text { consistent level for all } \\
\text { sessions. (Colour } \\
\text { temperature was set at } \\
6500 \mathrm{~K}) \text {. }\end{array}$ & CIELab & $\begin{array}{l}\text { The viewers' } \\
\text { shade-harmonizing } \\
\text { performance was significantly } \\
\text { improved using the computer } \\
\text { method compared to the } \\
\text { conventional one. }\end{array}$ \\
\hline Matis, 2000 & $\begin{array}{l}1 . \\
2 .\end{array}$ & $\begin{array}{l}\text { Digital camera. } \\
\text { Shade guide. }\end{array}$ & Human tooth & Non-specified & CIELab and $\Delta \mathrm{E}$ & $\begin{array}{l}\text { All three methods of evaluation } \\
\text { revealed a significant difference } \\
\text { in the tooth lightness. }\end{array}$ \\
\hline Matis, 2000 & $\begin{array}{l}1 . \\
2 .\end{array}$ & $\begin{array}{l}\text { Digital camera. } \\
\text { Shade guide. }\end{array}$ & Human tooth & Non-specified & CIELab & $\begin{array}{l}\text { This study suggests that when a } \\
\text { higher concentra-tion of } \\
\text { carbamide peroxide was used, } \\
\text { the further the lightness value } \\
\text { and } \Delta \mathrm{E} \text { altered. }\end{array}$ \\
\hline
\end{tabular}


Table 3. Cont

\begin{tabular}{|c|c|c|c|c|c|c|}
\hline Study & & $\begin{array}{l}\text { Method Used for } \\
\text { Shade Match }\end{array}$ & Materials Used & Type of Room Light & $\begin{array}{l}\text { Main } \\
\text { Outcome }\end{array}$ & Main Results \\
\hline $\begin{array}{l}\text { Gómez-Polo, } \\
2014\end{array}$ & $\begin{array}{l}1 . \\
2 .\end{array}$ & $\begin{array}{l}\text { 3DMaster Toothguide } \\
\text { (Vita-Zahnfabrik) } \\
\text { Easy-Shade Compact } \\
\text { (Vita-Zahnfabrik) } \\
\text { spectrophotometer }\end{array}$ & Human tooth & $\begin{array}{l}\text { Recordings were made } \\
\text { under fluorescent tubes } \\
\text { with daylight and an } \\
\text { intensity of } \\
1200-1500 \text { lux, in the } \\
\text { same room under } \\
\text { standardized lighting } \\
\text { conditions. }\end{array}$ & $\begin{array}{l}\text { Lightness } \\
\text { Chroma Hue }\end{array}$ & $\begin{array}{l}\text { This research showed } \\
\text { differences between the } \\
\text { measurement of color using the } \\
\text { spectrophotometry tool and the } \\
\text { visual shade } \\
\text { selection technique. }\end{array}$ \\
\hline Kröger, 2015 & $\begin{array}{l}1 . \\
2 .\end{array}$ & $\begin{array}{l}\text { Spectrophotometer } \\
\text { Visual color matching } \\
\text { using a shade guide }\end{array}$ & Human tooth & $\begin{array}{l}\text { A room with dimmed } \\
\text { fluorescent } \\
\text { ceiling light. }\end{array}$ & CIELab & $\begin{array}{l}\text { The spectrophotometer } \\
\text { provided higher reproducibility. }\end{array}$ \\
\hline Wang, 2014 & $\begin{array}{l}1 . \\
2 .\end{array}$ & $\begin{array}{l}\text { Spectrophotometer } \\
\text { Optimized and } \\
\text { Vitapan Classical } \\
\text { shade guide }\end{array}$ & Human tooth & Northern daylight & CIELab and $\Delta \mathrm{E}$ & $\begin{array}{l}\text { An optimized shade guide } \\
\text { improved the performance of } \\
\text { color selection }\end{array}$ \\
\hline He, 2019 & $\begin{array}{l}1 . \\
2 . \\
3 .\end{array}$ & $\begin{array}{l}\text { Non-polarized } \\
\text { photography } \\
\text { Cross-polarization } \\
\text { photography } \\
\text { Spectrophotometer }\end{array}$ & Human tooth & Non-specified & CIELab and $\Delta E$ & $\begin{array}{c}\text { Combining non-polarized } \\
\text { photography, cross-polarization } \\
\text { photography, and } \\
\text { spectrophotometer approaches } \\
\text { were considered reasonable for } \\
\text { shade matching. }\end{array}$ \\
\hline AlSaleh, 2012 & 1. & $\begin{array}{l}\text { VITA classical shade } \\
\text { guide (VITA } \\
\text { Zahnfabrik gmbh, } \\
\text { Bad Säckingen, } \\
\text { Germany) } \\
\text { Visual }\end{array}$ & Human tooth & $\begin{array}{l}\text { A light grey wall in a } \\
\text { room away from } \\
\text { all windows. }\end{array}$ & CIELab and $\Delta \mathrm{E}$ & $\begin{array}{l}\text { Analysis using the } \\
\text { spectrophotometric shade was } \\
\text { considered more accurate in } \\
\text { comparison to human } \\
\text { shade evaluation. }\end{array}$ \\
\hline $\begin{array}{l}\text { Baharin, } \\
2013\end{array}$ & $\begin{array}{l}1 . \\
2 .\end{array}$ & $\begin{array}{l}\text { Intraoral } \\
\text { spectrophotometer } \\
\text { machine } \\
\text { Visual }\end{array}$ & Human tooth & Non-specified & Accuracy & $\begin{array}{l}\text { This sudy revealed that for the } \\
\text { anterior tooth, the patient's } \\
\text { position, lighting condition and } \\
\text { number of readings acquired } \\
\text { does impact the outcome of } \\
\text { shade selection. }\end{array}$ \\
\hline $\begin{array}{l}\text { Bahannan, } \\
2014\end{array}$ & $\begin{array}{l}1 . \\
2 .\end{array}$ & $\begin{array}{l}\text { Visual method using } \\
\text { a Vita-3D Master } \\
\text { system } \\
\text { Spectrophotometer }\end{array}$ & Human tooth & Daylight & $\begin{array}{l}\text { Daylight } \\
\text { illuminator } \\
\text { (GTI Graphic } \\
\text { Technology, } \\
\text { NY, USA) }\end{array}$ & $\begin{array}{c}\text { The conventional visual method } \\
\text { was significantly inferior } \\
\text { compared to the shade } \\
\text { assessment method device. }\end{array}$ \\
\hline $\begin{array}{l}\text { Chitrarsu, } \\
2017\end{array}$ & $\begin{array}{l}1 . \\
2 .\end{array}$ & $\begin{array}{l}\text { Vita Toothguide } \\
\text { 3D-Master } \\
\text { Intraoral digital } \\
\text { spectrophotometer } \\
\text { (Vita Easyshade } \\
\text { Advance 4.0) }\end{array}$ & $\begin{array}{c}\text { Natural } \\
\text { dentitions }\end{array}$ & $\begin{array}{l}\text { Daylight, incandescent } \\
\text { light, LED, and } \\
\text { filtered LED. }\end{array}$ & CIELab & $\begin{array}{l}\text { Vita Toothguide 3D-Master } \\
\text { showed statistically important } \\
\text { variances in shade matching in } \\
\text { comparison to the intraoral } \\
\text { digital spectrophotometer. }\end{array}$ \\
\hline $\begin{array}{l}\text { Da Silva, } \\
2008\end{array}$ & $\begin{array}{l}1 . \\
2 .\end{array}$ & $\begin{array}{l}\text { Shade guide systems } \\
\text { Instrument-based } \\
\text { color matching using } \\
\text { a new spectro- } \\
\text { photometric system }\end{array}$ & $\begin{array}{l}\text { Tooth color for } \\
\text { anterior metal } \\
\text { ceramic } \\
\text { restorations }\end{array}$ & $\begin{array}{l}\text { Under daylight and } \\
\text { color temperature of } \\
6500^{\circ} \mathrm{K} \text {. }\end{array}$ & $\Delta \mathrm{E}$ & $\begin{array}{l}\text { For anterior metal ceramic } \\
\text { restorations, using a } \\
\text { spectrophotometric method is } \\
\text { an effective device for imitating } \\
\text { and communicating the color of } \\
\text { the tooth. }\end{array}$ \\
\hline Hein, 2016 & 1. & $\begin{array}{l}\text { A digital single-lens } \\
\text { reflex camera. }\end{array}$ & $\begin{array}{l}\text { Extracted human } \\
\text { teeth }\end{array}$ & Non-specified & CIELab and $\Delta \mathrm{E}$ & $\begin{array}{l}\text { The use of a white balance } \\
\text { reference card with } \\
\text { acknowledged color } \\
\text { coordinates can be suggested } \\
\text { when diffusers are used for } \\
\text { dental photography. }\end{array}$ \\
\hline
\end{tabular}


Table 3. Cont

\begin{tabular}{|c|c|c|c|c|c|c|}
\hline Study & & $\begin{array}{l}\text { Method Used for } \\
\text { Shade Match }\end{array}$ & Materials Used & Type of Room Light & $\begin{array}{l}\text { Main } \\
\text { Outcome }\end{array}$ & Main Results \\
\hline $\begin{array}{l}\text { Miyajiwala, } \\
2017\end{array}$ & $\begin{array}{l}1 . \\
2 . \\
3 .\end{array}$ & $\begin{array}{l}\text { Visual method } \\
\text { Spectrophotometer } \\
\text { Digital photography }\end{array}$ & Human tooth & Daylight & CIELab & $\begin{array}{l}\text { Clinically, for shade selection, } \\
\text { the use of the digital } \\
\text { photography method can } \\
\text { appear as a viable alternative to } \\
\text { the use of spectro- } \\
\text { photometric method. }\end{array}$ \\
\hline Li, 2007 & $\begin{array}{l}1 . \\
2 .\end{array}$ & $\begin{array}{l}\text { Shade guide } \\
\text { Shofu ShadeEye } \\
\text { NCC colorimeter }\end{array}$ & Human tooth & Northern daylight & CIELab & $\begin{array}{l}\text { The consistency of shade } \\
\text { matching cannot be guaranteed } \\
\text { by either the visual method or } \\
\text { the colorimeter approach. }\end{array}$ \\
\hline $\begin{array}{l}\text { Pimentel, } \\
2014\end{array}$ & $\begin{array}{l}1 . \\
2 .\end{array}$ & $\begin{array}{l}\text { Visual (classic } \\
\text { shade guide) } \\
\text { Instrumental } \\
\text { methods } \\
\text { (spectrophotometer) }\end{array}$ & Natural tooth & Controlled illumination & Accuracy & $\begin{array}{l}\text { Shade match using the } \\
\text { instrumental method presented } \\
\text { more agreement than shade } \\
\text { match using the visual method. }\end{array}$ \\
\hline Okubo, 1998 & $\begin{array}{l}1 . \\
2 .\end{array}$ & $\begin{array}{l}\text { Vita Lumin shade } \\
\text { guide teeth } \\
\text { A computerized } \\
\text { colorimeter }\end{array}$ & $\begin{array}{l}\text { Ceramic shade } \\
\text { guide teeth }\end{array}$ & Northern daylight & CIELab and $\Delta \mathrm{E}$ & $\begin{array}{l}\text { Color matching using the visual } \\
\text { process is unpredictable. } \\
\text { However, instrumental } \\
\text { measurement of tooth color } \\
\text { would deliver objective } \\
\text { measured data to match the } \\
\text { color of the natural teeth. }\end{array}$ \\
\hline Olms, 2013 & 1. & $\begin{array}{l}\text { VITA Easyshade } \\
\text { spectrophotometer }\end{array}$ & Ceramic veneer & $\begin{array}{c}\text { Ceiling lighting } \\
\text { (Philips Master TLD } 36 \\
\text { W) and a dental lamp } \\
\text { (KaVo Dental GmbH, } \\
\text { Germany). }\end{array}$ & CIELab & $\begin{array}{l}\text { This study confirmed that } \\
\text { worthy outcomes in terms of } \\
\text { the repeatability and precision } \\
\text { were obtained with the help of } \\
\text { the VITA Easyshade } \\
\text { measurements. }\end{array}$ \\
\hline Paul, 2002 & 1. & $\begin{array}{l}\text { Spectrophotometric } \\
\text { assessment of tooth } \\
\text { color visual } \\
\text { determination }\end{array}$ & Human tooth & Light source (6500 K). & $\Delta \mathrm{E}$ & $\begin{array}{l}\text { Human shade assessment is } \\
\text { less accurate and less } \\
\text { reproducible when compared to } \\
\text { spectrophotometric shade } \\
\text { evaluation. }\end{array}$ \\
\hline $\begin{array}{l}\text { Schropp, } \\
2008\end{array}$ & 2. & $\begin{array}{l}\text { Digital photographs } \\
\text { with graphic } \\
\text { computer software } \\
\text { Conventional visual } \\
\text { matching }\end{array}$ & Phantom head & $\begin{array}{c}\text { Daylight lamps with a } \\
\text { color temperature of } \\
4800 \mathrm{~K} .\end{array}$ & CIE LCh & $\begin{array}{l}\text { Digital photographs and } \\
\text { computer software were } \\
\text { significantly more trustworthy } \\
\text { than conventional visual } \\
\text { approach for shade- } \\
\text { matching analysis. }\end{array}$ \\
\hline Tung, 2010 & 1. & $\begin{array}{l}\text { Digital camera in } \\
\text { both automatic white } \\
\text { balance }(\mathrm{AWB}) \text { and } \\
\text { custom white balance } \\
\text { (CWB) under either } \\
\text { light-emitting diode } \\
\text { (LED) or electronic } \\
\text { ring flash }\end{array}$ & Ceramic disks & $\begin{array}{l}\text { The background } \\
\text { lighting in the room } \\
\text { was subdued and } \\
\text { maintained at a } \\
\text { constant level during } \\
\text { the entire experiment. }\end{array}$ & CIELab & $\begin{array}{l}\text { Digital images were more } \\
\text { influenced by the illuminants } \\
\text { and camera's white balance } \\
\text { setups when testing the } \\
\text { reliability of color match. }\end{array}$ \\
\hline Wee, 2002 & $\begin{array}{l}1 . \\
2 . \\
3 .\end{array}$ & $\begin{array}{l}\text { Vita Lumin/Vita } \\
\text { VMK } 68 \\
\text { Vitapan 3D-Master/ } \\
\text { Vita Omega } 900 \\
\text { Shofu ShadeEye- } \\
\text { EX/Vintage Halo }\end{array}$ & Dental porcelain & $\begin{array}{l}\text { Color-corrected } \\
\text { D65 lighting. }\end{array}$ & CIELab & $\begin{array}{l}\text { The largest mean } \Delta \mathrm{E} \text { was noted } \\
\text { for the Vitapan } 3 \mathrm{D} \text {-Master } \\
\text { system, which was considerably } \\
\text { distinct from the Vita Lumin } \\
\text { and Shofu ShadeEye systems. }\end{array}$ \\
\hline
\end{tabular}


Table 3. Cont

\begin{tabular}{|c|c|c|c|c|c|c|}
\hline Study & & $\begin{array}{l}\text { Method Used for } \\
\text { Shade Match }\end{array}$ & Materials Used & Type of Room Light & $\begin{array}{l}\text { Main } \\
\text { Outcome }\end{array}$ & Main Results \\
\hline $\begin{array}{l}\text { Yamanel, } \\
2010\end{array}$ & $\begin{array}{l}1 . \\
2 .\end{array}$ & $\begin{array}{l}\text { Digital imaging } \\
\text { Colorimeter }\end{array}$ & $\begin{array}{l}\text { Composite resin } \\
\text { shade guides }\end{array}$ & $\begin{array}{l}\text { Two } 6500-\mathrm{K} \text { fluorescent } \\
\text { tubes were combined } \\
\text { with two } 2700 \mathrm{~K} \\
\text { fluorescent tubes. }\end{array}$ & CIELab & $\begin{array}{l}\text { The mean } \Delta \mathrm{E} \text { values verified a } \\
\text { statistically significant } \\
\text { difference with the colorimeter } \\
\text { method. However, there was no } \\
\text { significant change when using } \\
\text { the digital imaging approach. }\end{array}$ \\
\hline Yilmaz, 2010 & $\begin{array}{l}1 . \\
2 .\end{array}$ & $\begin{array}{l}\text { Intraoral colorimeter } \\
\text { (shadeeye NCC) } \\
\text { Visual shade } \\
\text { determination }\end{array}$ & Metal ceramic & Non-specified & CIELab & $\begin{array}{l}\text { Color imitation using } \\
\text { instrumental shade method for } \\
\text { the specimens made of metal } \\
\text { ceramic was less accurate when } \\
\text { compared to the visual } \\
\text { shade approach. }\end{array}$ \\
\hline $\begin{array}{l}\text { Lakhanpal, } \\
2016\end{array}$ & $\begin{array}{l}1 . \\
2 . \\
3 .\end{array}$ & $\begin{array}{l}\text { Spectrophotometer } \\
\text { Digital camera } \\
\text { Digital camera with a } \\
\text { polarizer }\end{array}$ & $\begin{array}{c}\text { Extracted } \\
\text { non-carious } \\
\text { premolars }\end{array}$ & Dark room setup. & CIELab & $\begin{array}{l}\text { A statistically important } \\
\text { association was found to exist } \\
\text { with the spectrophotometer and } \\
\text { the polarization dental imaging } \\
\text { modality for all CIE Lab } \\
\text { color coordinates }\end{array}$ \\
\hline Kelkar, 2020 & $\begin{array}{l}1 . \\
2 .\end{array}$ & $\begin{array}{l}\text { Canon 5D camera } \\
\text { with ISO } 200 \\
\text { Two VITAPAN classi- } \\
\text { cal shade guides }\end{array}$ & VITAPAN & Daylight & $\begin{array}{l}\text { Individual } \\
\text { Matching } \\
\text { Ability of each } \\
\text { Observer }\end{array}$ & $\begin{array}{l}\text { For obtaining an aesthetic } \\
\text { outcome, the use of digital } \\
\text { photographic approach was } \\
\text { considered most accurate } \\
\text { among the three shade } \\
\text { selection approaches. }\end{array}$ \\
\hline
\end{tabular}

The meta-analysis of the quantitative data indicates that $(\Delta \mathrm{E})$ between restorations where the shade matching was performed with the conventional method was greater than those where the shade matching was performed with the computerized methods $(p=0.007)$. According to the subgroup analysis, only the use of digital photographs for shade matching showed a reduction in $(\Delta \mathrm{E})(p<0.001)$, while the use of a spectrophotometer had no advantages over the use of visual shade guide tabs $(p=0.57)$ (Figure 2$)$.

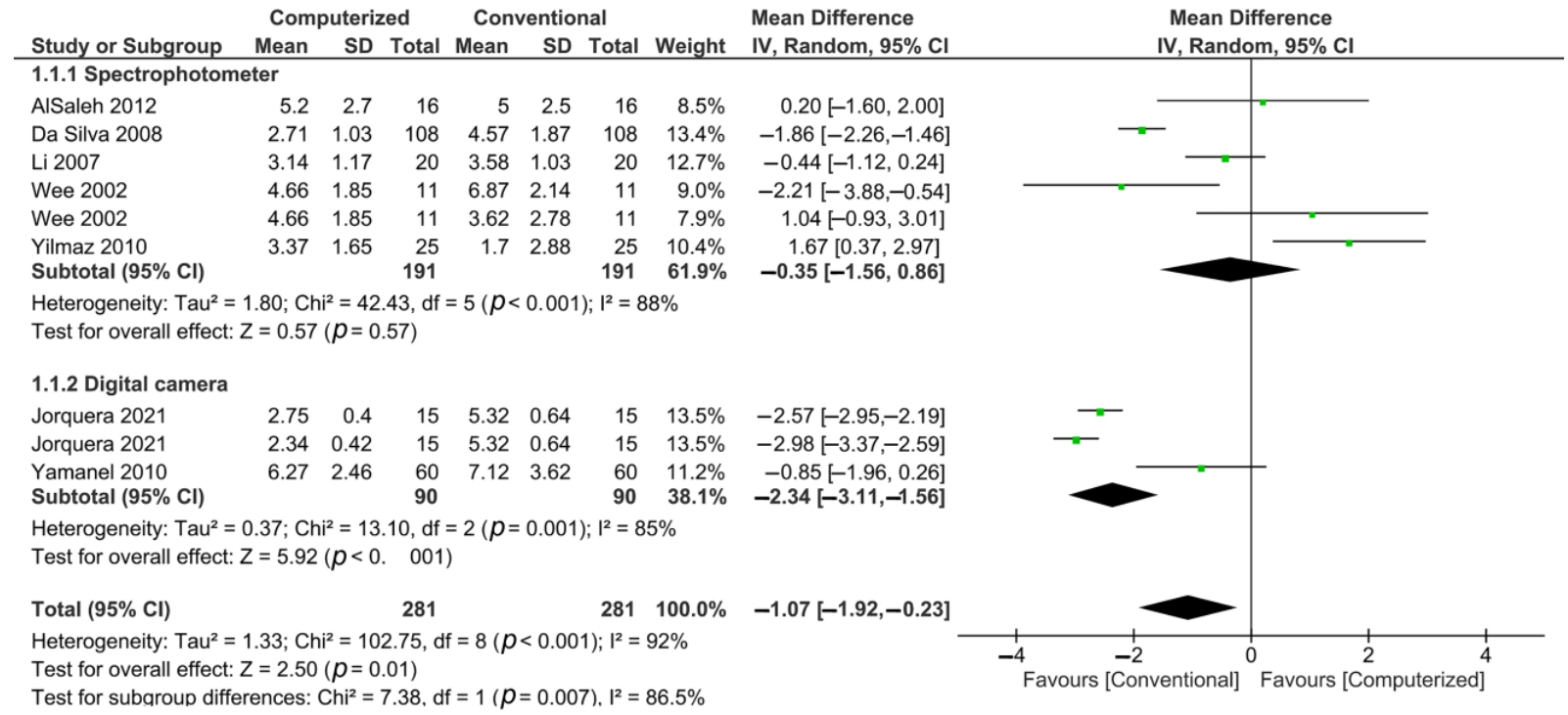

Figure 2. Forest plot of the quantitative analysis.

On the other hand, for the qualitative analysis, global analysis proved that incorrect shade matching was higher when the conventional method using shade guide tabs was used $(p<0.001)$, irrespective of whether a spectrophotometer or a digital camera was used $(p<0.001)$ (Figure 3). 


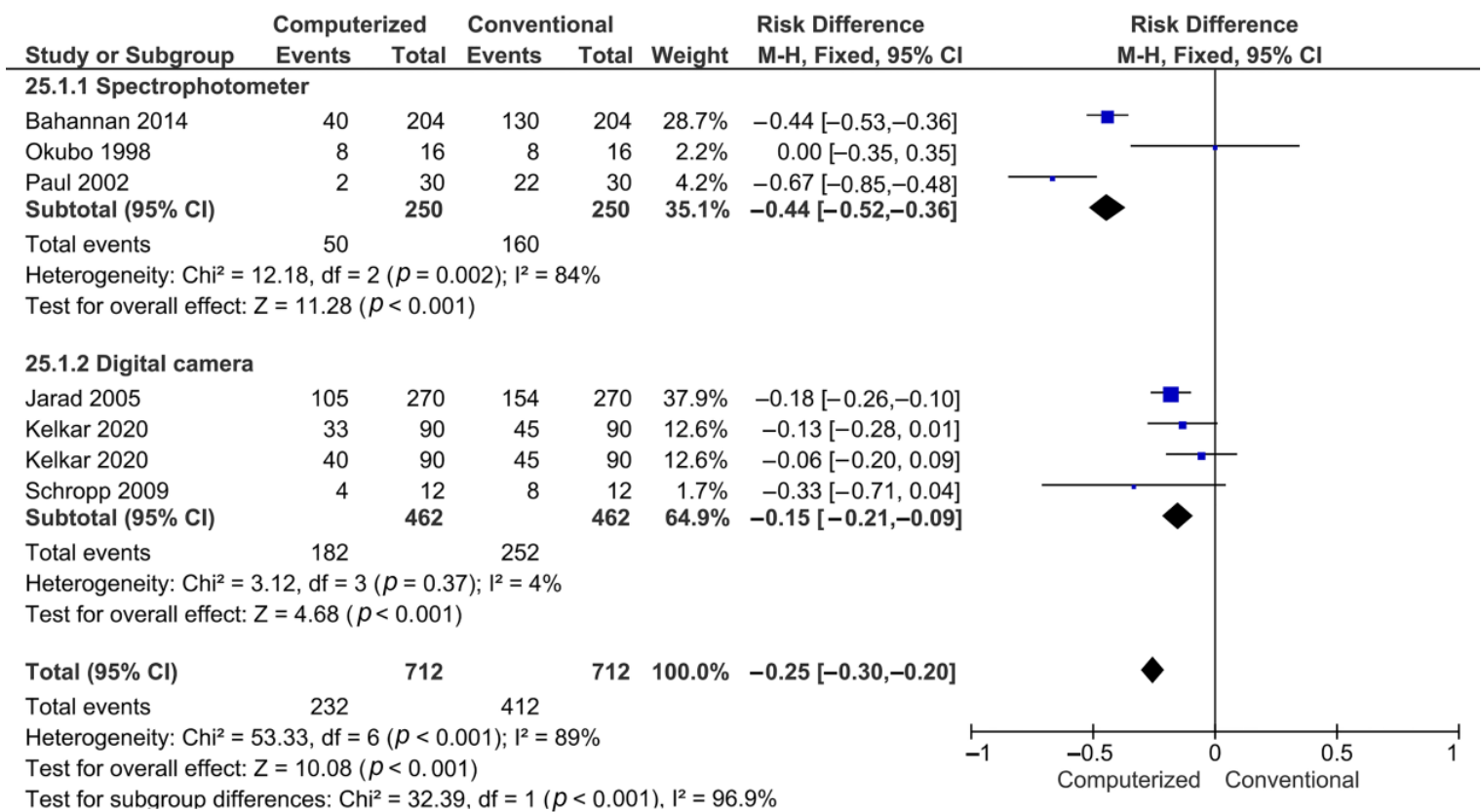

Figure 3. Forest plot of the qualitative analysis.

Studying the methodological quality assessment parameters, most of the manuscripts involved were counted as having a medium risk of bias (Table 4 and Figure 4). However, several studies analyzed failed to account for the sample size calculation, single operator, and operator blinded parameters. Regarding the clinical studies, most of them were categorized as having a high risk of bias, since most of them failed to avoid performance and detection bias, reporting bias, bias due to incomplete data, and other bias.

Table 4. Qualitative synthesis for in vitro articles.

\begin{tabular}{|c|c|c|c|c|c|c|c|}
\hline Study & $\begin{array}{c}\text { Specimen } \\
\text { Randomization }\end{array}$ & $\begin{array}{l}\text { Single } \\
\text { Operator }\end{array}$ & $\begin{array}{l}\text { Operator } \\
\text { Blinded }\end{array}$ & $\begin{array}{l}\text { Control } \\
\text { Group }\end{array}$ & $\begin{array}{l}\text { Standardized } \\
\text { Specimens }\end{array}$ & $\begin{array}{l}\text { Sample Size } \\
\text { Calculation }\end{array}$ & Risk of Bias \\
\hline Jarad, 2003 & YES & YES & $\mathrm{NO}$ & YES & YES & $\mathrm{NO}$ & Medium \\
\hline Kröger, 2015 & YES & $\mathrm{NO}$ & NO & YES & YES & YES & Medium \\
\hline He, 2019 & NO & $\mathrm{NO}$ & NO & YES & YES & NO & High \\
\hline Hein, 2016 & YES & $\mathrm{NO}$ & NO & YES & YES & NO & Medium \\
\hline Schropp, 2008 & $\mathrm{NO}$ & $\mathrm{NO}$ & YES & YES & YES & YES & Medium \\
\hline Tung, 2010 & YES & $\mathrm{NO}$ & YES & YES & YES & NO & Medium \\
\hline Wee, 2002 & YES & $\mathrm{NO}$ & $\mathrm{NO}$ & YES & YES & $\mathrm{NO}$ & Medium \\
\hline Yamanel, 2010 & $\mathrm{NO}$ & $\mathrm{NO}$ & $\mathrm{NO}$ & YES & YES & $\mathrm{NO}$ & High \\
\hline Yilmaz, 2010 & YES & $\mathrm{NO}$ & YES & YES & YES & YES & Low \\
\hline Lakhanpal, 2016 & $\mathrm{NO}$ & $\mathrm{NO}$ & NO & YES & YES & NO & High \\
\hline Kelkar, 2020 & YES & $\mathrm{NO}$ & NO & YES & YES & YES & Medium \\
\hline
\end{tabular}

Random sequence generation (selection bias)

Blinding of participants and personnel (performance bias)

Incomplete outcome data (attrition bias)

Selective reporting (reporting bias)

Other bias

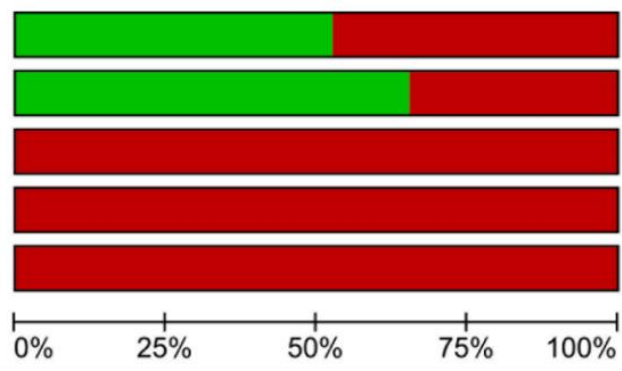

Low risk of bias

Unclear risk of bias

High risk of bias

Figure 4. Qualitative synthesis for clinical trials. 


\section{Discussion}

This systematic review and meta-analysis were directed to compare different computerized methods with the visual shade guides for shade matching. The overall findings revealed that the color differences of restorations performed using a visual shade guide were higher than with digital photography. In addition, the proportion of incorrect shade matching was higher when the visual shade guide tabs were used. Considering this, the null hypothesis stating that both digital and visual shade matching of the tooth were comparable was rejected.

It should be noted that shade matching in esthetic restorations was a key factor in the attainment of a perfect tooth color $[47,48]$. Furthermore, color mismatch in ceramic restorations can be very stressful for both clinicians and patients [49]. In this sense, the combination of visual and digital techniques has been suggested for a proper tooth color matching [35]. The visual technique consists of the use of shade guides to acquire the closest match to a natural tooth [5,7]. This way is subjective, and its precision is affected by several factors including experience, sex, and the need for training of the spectator, in addition to the tooth shade and light source [50-52]. Dental technicians should rely on a color match approach when operating only from an instruction based on a shade guide [53]. For this reason, the digital technique has been deemed more reliable than the visual technique alone [7]. Digital images achieved imperative data about tooth color crossways and the shape, surface, and characteristic features that the dental technician needs [35]. This could be explained by the improvement in communication and color selection [54]. Whenever feasible, both techniques should be combined [5,54].

In addition, mobile dental photography (MDP) seems to be favorable in terms of software applications, cost effectiveness, enhanced functionalities, and high-resolution photographs, which makes it suitable for acquiring color references $[54,55]$. The use of a smartphone with the help of the cross-polarizing filter of the smile Lite MDP allowed users to compare and evaluate the color matching amongst shade tabs $[54,56,57]$.

One should bear in mind that by using this filter, the intrinsic shade variations of natural teeth for shade analysis might be revealed. In addition, it can help remove unwanted reflections and diffuse light created by the flash which can obscure features in the dental teeth, and hence produce issues in communication with the dental technician $[18,21]$. Consequently, referring to the results of Sampaio et al., [27] the use of cross-polarizing filters was the most standardized method for both communication and color assessment. It is important to mention that non-polarized tooth color images offered numerous glare designs on the surface of the tooth, which could be affected by the uneven distribution of saliva on tooth surfaces or roughness. These produce artifacts in tooth color image analysis. In juxtapose, a lack of glaring patterns was observed in the cross-polarized tooth color images [32].

It should be emphasized that $(\Delta \mathrm{E})$ obtained by two measurements of the same object can be interpreted as acceptable or perceptible to the human eyes [58].

Acceptability means the agreement of the color of the restoration, while perceptibility indicates the detection of the $(\Delta \mathrm{E})$ between both the tooth and the adjacent restoration [59]. A previous study suggested that a clinically acceptable level of visual perception was possible when $(\Delta \mathrm{E}=3.7)$ in the CIELab system [58]. This system contains three coordinates: $\mathrm{L}^{*}$ which refers to the luminosity of the object and fluctuates from black to white; $\mathrm{a}^{*}$ which indicates the chroma in the green-red axis; and the $b^{*}$ which represents the chroma in the axis of blue-yellow [60]. Each color has a numerical value, delivering an objective description [27]. This might clarify the outcomes of this analysis obtained by a visual technique, as the preceding paper displayed that a non-acceptable restoration match was obtained with a higher $(\Delta E)$ [35]. In this respect, clinicians should consider the digital technique when performing the shade-matching process. Digital images gave significant evidence about tooth color crossways, the surface, shape, and details that the dental laboratory technician needs $[61,62]$. Indeed, this could be possible when using a standardized light correcting device, thus helping to achieve a better result [59]. Both smartphones and digital 
cameras can promote a correct white balance by using a grey reference card with specific color coordinates to supply the color of the images in an accurate way $[19,27,35]$. This was considered successful in compensating the changes in tooth color triggered by various diffuser materials [19].

Additionally, a cross-polarizing filter might be assessed to eliminate specular reflections which can modify an image and lead to an imperfect analysis [27]. Hence, the use of less expensive instruments such as smartphones may enhance communication and help replicate the color in an easy, fast, and accurate way.

The percentage of dentists trusting the use of their smartphone cameras instead of professional DSLR cameras is rising exponentially because of the former's easy access and manipulation [63].

The findings of this analysis should be reflected with caution, since not all smartphones and digital cameras available on the market were tested. In addition, the evidence should be directed towards different light source devices which can facilitate communication between the dentist, patient, and the lab technician. Future research needs to consist of guided, principally randomized controlled clinical trials, with the aim of affording better vision into the performance of several computerized approaches to color-shade-matching success. Moreover, different spectrophotometers are already available in the market, and further studies testing these devices can provide a better understanding of color matching between the tooth and the restoration in an affordable way.

\section{Conclusions}

The use of digital photography and spectrophotometric measurements led to fewer color differences and less incorrect shade matching than conventional methods using color shade tabs.

Supplementary Materials: The following supporting information can be downloaded at: https: / / www.mdpi.com/article/10.3390/ma15020468/s1, Table S1: Search strategy used in SCOPUS; Table S2: Search strategy used in ISI Web of Science; Table S3: Search strategy used in the Cochrane Library; Table S4: Search strategy used in EMBASE.

Author Contributions: Conceptualization, L.H. and R.B.; search strategy, M.L.-S. and A.J.M.-Á.; literature search, L.H. and R.B.; study selection, L.H. and R.B.; data extraction, Y.H., G.J., D.M., M.L.-S. and T.A.; quality assessment, L.H. and R.B.; software, L.H., R.B. and C.E.C.-S.; validation, A.J.M.-Á., G.J., D.M., M.L.-S. and T.A.; formal analysis, L.H., M.L.-S., R.B. and C.E.C.-S.; investigation, M.L.-S., A.J.M.-Á., G.J., D.M., L.H., R.B. and T.A.; resources, G.J., N.K., L.H., M.Z., N.J., Y.H. and R.B.; data curation, L.H., R.B., M.L.-S., N.K. and C.E.C.-S.; writing-original draft preparation, L.H., R.B., N.K., M.L.-S., C.E.C.-S. and Y.H.; writing-review and editing, Y.H., L.H., M.Z., N.K., N.J., C.E.C.-S. and R.B.; visualization, L.H. and R.B.; supervision, L.H.; project administration, L.H. All authors have read and agreed to the published version of the manuscript.

Funding: This research received no external funding.

Data Availability Statement: The data that help the outcomes of this analysis are accessible from the corresponding authors upon reasonable request.

Acknowledgments: Authors Louis Hardan and Rim Bourgi would like to thank the Saint-Joseph University of Beirut, Lebanon. Furthermore, the reviewers would also thank the University of Hidalgo State, Mexico, the Medical University of Lodz, and the University of Strasbourg for accompanying this research.

Conflicts of Interest: The authors state no conflict of interest.

\section{References}

1. Gasparik, C.; Grecu, A.G.; Culic, B.; Badea, M.E.; Dudea, D. Shade-matching Performance Using a New Light-correcting Device. J. Esthet. Res. Dent. 2015, 27, 285-292. [CrossRef] [PubMed]

2. Öngül, D.; Şermet, B.; Balkaya, M.C. Visual and instrumental evaluation of color match ability of 2 shade guides on a ceramic system. J. Prosthet. Dent. 2012, 108, 9-14. [CrossRef] 
3. Horn, D.J.; Bulan-Brady, J.; Hicks, M.L. Sphere Spectrophotometer versus Human Evaluation of Tooth Shade. J. End. 1998, 24, 786-790. [CrossRef]

4. Takatsui, F.; de Andrade, M.F.; Neisser, M.P.; Barros, L.A.B.; de Loffredo, L.C.M. CIE L* A* B*: Comparison of Digital Images Obtained Photographically by Manual and Automatic Modes. Braz. Oral Res. 2012, 26, 578-583. [CrossRef]

5. $\quad$ van der Burgt, T.P.; ten Bosch, J.J.; Borsboom, P.C.F.; Kortsmit, W. A Comparison of New and Conventional Methods for Quantification of Tooth Color. J. Prosthet. Dent. 1990, 63, 155-162. [CrossRef]

6. Gokce, H.S.; Piskin, B.; Ceyhan, D.; Gokce, S.M.; Arisan, V. Shade Matching Performance of Normal and Color Vision-Deficient Dental Professionals with Standard Daylight and Tungsten Illuminants. J. Prosthet. Dent. 2010, 103, 139-147. [CrossRef]

7. Preethi Suganya, S.; Manimaran, P.; Saisadan, D.; Dhinesh Kumar, C.; Abirami, D.; Monnica, V. Evaluation of Shade Selection with Digital and Visual Methods. J. Pharm. Bioallied. Sci. 2020, 12, 319-323. [CrossRef] [PubMed]

8. Tabatabaian, F.; Beyabanaki, E.; Alirezaei, P.; Epakchi, S. Visual and digital tooth shade selection methods, related effective factors and conditions, and their accuracy and precision: A literature review. J. Esthet. Restor. Dent. 2021, 33, 1084-1104. [CrossRef]

9. Brewer, J.D.; Wee, A.; Seghi, R. Advances in Color Matching. Dent. Clin. 2004, 48, 341-358. [CrossRef] [PubMed]

10. Douglas, R.D.; Przybylska, M. Predicting Porcelain Thickness Required for Dental Shade Matches. J. Prosthet. Dent. 1999, 82, 143-149. [CrossRef]

11. Blaes, J. Today's Technology Improves the Shade-Matching Problems of Yesterday. J. Indiana Dent. Assoc. 2002, 81, 17-19. [PubMed]

12. Czigola, A.; Róth, I.; Vitai, V.; Fehér, D.; Hermann, P.; Borbély, J. Comparing the effectiveness of shade measurement by intraoral scanner, digital spectrophotometer, and visual shade assessment. J. Esthet. Restor. Dent. 2021, 33, 1166-1174. [CrossRef]

13. Dietschi, D.; Fahl, N., Jr. Shading concepts and layering techniques to master direct anterior composite restorations: An update. Br. Dent. J. 2016, 221, 765-771. [CrossRef]

14. Page, M.; McKenzie, J.; Bossuyt, P.; Boutron, I.; Hoffman, T.; Mulrow, C.; Shamseer, L.; Tetzlaff, J.; Akl, E.; Brennan, S.; et al. The PRISMA 2020 Statement: An Updated Guideline for Reporting Systematic Reviews. BMJ 2021, 372, n71. [CrossRef]

15. Bourgi, R.; Hardan, L.; Rivera-Gonzaga, A.; Cuevas-Suárez, C.E. Effect of Warm-Air Stream for Solvent Evaporation on Bond Strength of Adhesive Systems: A Systematic Review and Meta-Analysis of in Vitro Studies. Int. J. Adhes. Adhes. 2021, 105, 102794. [CrossRef]

16. Matis, B.A.; Wang, Y.; Jiang, T.; Eckert, G.J. Extended At-Home Bleaching of Tetracycline-Stained Teeth with Different Concentrations of Carbamide Peroxide. Quintessence Int. 2002, 33, 645-655. [PubMed]

17. Matis, B.A.; Mousa, H.N.; Cochran, M.A.; Eckert, G.J. Clinical Evaluation of Bleaching Agents of Different Concentrations. Quintessence Int. 2000, 31, 303-310. [PubMed]

18. Mahn, E.; Tortora, S.C.; Olate, B.; Cacciuttolo, F.; Kernitsky, J.; Jorquera, G. Comparison of Visual Analog Shade Matching, a Digital Visual Method with a Cross-Polarized Light Filter, and a Spectrophotometer for Dental Color Matching. J. Prosthet. Dent. 2021, 125, 511-516. [CrossRef]

19. Hein, S.; Zangl, M. The Use of a Standardized Gray Reference Card in Dental Photography to Correct the Effects of Five Commonly Used Diffusers on the Color of 40 Extracted Human Teeth. Int. J. Esthet. Dent. 2016, 11, 246-259.

20. Kröger, E.; Matz, S.; Dekiff, M.; Tran, B.L.; Figgener, L.; Dirksen, D. In Vitro Comparison of Instrumental and Visual Tooth Shade Determination under Different Illuminants. J. Prosthet. Dent. 2015, 114, 848-855. [CrossRef]

21. He, W.H.; Park, C.J.; Byun, S.; Tan, D.; Lin, C.Y.; Chee, W. Evaluating the Relationship between Tooth Color and Enamel Thickness, Using Twin Flash Photography, Cross-Polarization Photography, and Spectrophotometer. J. Esthet. Dent. 2020, 32, 91-101. [CrossRef]

22. Matis, B.A.; Wang, Y.; Jiang, T.; Eckert, G.J. A Clinical Evaluation of a Bleaching Agent Used With and Without Reservoirs. Oper. Dent. 2002, 27, 5-11. [PubMed]

23. Miyajiwala, J.; Kheur, M.; Patankar, A.; Lakha, T. Comparison of Photographic and Conventional Methods for Tooth Shade Selection: A Clinical Evaluation. J. Indian Prosthodont. Soc. 2017, 17, 273-281.

24. Wang, P.; Wei, J.; Li, Q.; Wang, Y. Evaluation of an optimized shade guide made from porcelain powder mixtures. J. Prosthet. Dent. 2014, 112, 1553-1558. [CrossRef] [PubMed]

25. Tung, O.H.; Lai, Y.L.; Ho, Y.C.; Chou, I.C.; Lee, S.Y. Development of Digital Shade Guides for Color Assessment Using a Digital Camera with Ring Flashes. Clin. Oral Investig. 2011, 15, 49-56. [CrossRef]

26. Olms, C.; Setz, J.M. The Repeatability of Digital Shade Measurement-a Clinical Study. Clin. Oral Investig. 2013, 17, 1161-1166. [CrossRef]

27. Sampaio, C.S.; Atria, P.J.; Hirata, R.; Jorquera, G. Variability of Color Matching with Different Digital Photography Techniques and a Gray Reference Card. J. Prosthet. Dent. 2019, 121, 333-339. [CrossRef]

28. Pimentel, W.; Tiossi, R. Comparison between Visual and Instrumental Methods for Natural Tooth Shade Matching. Gen. Dent. 2014, 62, 47-49.

29. Gómez-Polo, C.; Gómez-Polo, M.; Celemin-Viñuela, A.; Martínez Vázquez De Parga, J.A. Differences between the Human Eye and the Spectrophotometer in the Shade Matching of Tooth Colour. J. Dent. 2014, 42, 742-745. [CrossRef] [PubMed]

30. Matis, B.; Nousa, H.N.; Cochran, M.A.; Eckert, G. Clinical Evaluation of In-Office and at-Home Bleaching Treatments Dental Trauma View Project Polymerization Pattern within a Resin-Based Composite View Project. Esth. Dent. 2000, 31, 303-310. 
31. Chitrarsu, V.K.; Chidambaranathan, A.S.; Balasubramaniam, M. Analysis of Shade Matching in Natural Dentitions Using Intraoral Digital Spectrophotometer in LED and Filtered LED Light Sources. J. Prosth. 2019, 28, e68-e73. [CrossRef]

32. Lakhanpal, S.; Neelima, M.S. Accuracy of Three Shade-Matching Devices in Replicating the Shade of Metal Ceramic Restorations: An in Vitro Study. J. Contemp. Dent. Pract. 2016, 17, 1003-1008. [CrossRef]

33. Baharin, S.A.; Tey, Y.D.; Tan, W.J. Anterior Tooth Shade Selection Procedure: Influence of Light Sources and Patient's Position. Sains Malays. 2013, 42, 7-11.

34. Kelkar, K.C.; Dogra, E.S.; Bhat, V.; Prasad, D.K.; Hegde, C. A Comparison between Visual, Digital Photography and Polarizing Filter Photography for Shade Selection. Indian J. Dent. Res. 2020, 31, 712.

35. Jorquera, G.J.; Atria, P.J.; Galán, M.; Feureisen, J.; Imbarak, M.; Kernitsky, J.; Cacciuttolo, F.; Hirata, R.; Sampaio, C.S. A Comparison of Ceramic Crown Color Difference between Different Shade Selection Methods: Visual, Digital Camera, and Smartphone. J. Prosthet. Dent. 2021. [CrossRef]

36. Yamanel, K.; Caglar, A.; Özcan, M.; Gulsah, K.; Bagis, B. Assessment of Color Parameters of Composite Resin Shade Guides Using Digital Imaging versus Colorimeter. J. Esthet. Dent. 2010, 22, 379-388. [CrossRef] [PubMed]

37. Li, Q.; Wang, Y.N. Comparison of Shade Matching by Visual Observation and an Intraoral Dental Colorimeter. J. Oral Rehabil. 2007, 34, 848-854. [CrossRef] [PubMed]

38. Alsaleh, S.; Labban, M.; Alhariri, M.; Tashkandi, E. Evaluation of Self Shade Matching Ability of Dental Students Using Visual and Instrumental Means. J. Dent. 2012, 40, e82-e87. [CrossRef]

39. da Silva, J.D.; Park, S.E.; Weber, H.-P.; Dent, M.; Ishikawa-Nagai, S.; Silva, D. Clinical Performance of a Newly Developed Spectrophotometric System on Tooth Color Reproduction. J. Prosthet. Dent. 2008, 99, 361-368. [CrossRef]

40. Yilmaz, B.; Karaagaclioglu, L. In Vitro Evaluation of Color Replication of Metal Ceramic Specimens Using Visual and Instrumental Color Determinations. J. Prosthet. Dent. 2010, 105, 21-27. [CrossRef]

41. Paul, S.; Peter, A.; Pietrobon, N.; Hämmerle, C.H.F. Visual and Spectrophotometric Shade Analysis of Human Teeth. J. Dent. Res. 2002, 81, 578-582. [CrossRef]

42. Schropp, L. Shade Matching Assisted by Digital Photography and Computer Software. J. Prosthodont. 2009, 18, 235-241. [CrossRef] [PubMed]

43. Jarad, F.D.; Russell, M.D.; Moss, B.W. The Use of Digital Imaging for Colour Matching and Communication in Restorative Dentistry. Br. Dent. J. 2005, 199, 43-49. [CrossRef]

44. Bahannan, S.A. Shade Matching Quality among Dental Students Using Visual and Instrumental Methods. J. Dent. 2014, 42, 48-52. [CrossRef]

45. Okubo, S.R.; Kanawati, A.; Richards, M.W.; Childressd, S. Evaluation of Visual and Instrument Shade Matching. J. Prosthet. Dent. 1998, 80, 642-648. [CrossRef]

46. Wee, A.G.; Monaghan, P.; Johnston, W.M. Variation in Color between Intended Matched Shade and Fabricated Shade of Dental Porcelain. J. Prosthet. Dent. 2002, 87, 657-666. [CrossRef]

47. Alghazali, N.; Burnside, G.; Moallem, M.; Smith, P.; Preston, A.; Jarad, F.D. Assessment of Perceptibility and Acceptability of Color Difference of Denture Teeth. J. Dent. 2012, 40, e10-e17. [CrossRef] [PubMed]

48. Bergman, B.; Nilson, H.; Andersson, M. A Longitudinal Clinical Study of Procera Ceramic-Veneered Titanium Copings. Int. J. Prosthodont. 1999, 12, 135-139.

49. Paolone, G.; Orsini, G.; Manauta, J.; Devoto, W.; Putignano, A. Composite shade guides and color matching. Int. J. Esthet Dent. 2014, 9, 164-182.

50. Haddad, H.J.; Jakstat, H.A.; Arnetzl, G.; Borbely, J.; Vichi, A.; Dumfahrt, H.; Renault, P.; Corcodel, N.; Pohlen, B.; Marada, G.; et al. Does gender and experience influence shade matching quality? J. Dent. 2009, 37, 40-44. [CrossRef]

51. Tam, W.K.; Lee, H.J. Dental Shade Matching Using a Digital Camera. J. Dent. 2012, 40, e3-e10. [CrossRef]

52. Clary, J.A.; Ontiveros, J.C.; Cron, S.G.; Paravina, R.D. Influence of Light Source, Polarization, Education, and Training on Shade Matching Quality. J. Prosthet. Dent. 2016, 116, 91-97. [CrossRef]

53. Alomari, M.; Chadwick, R.G. Factors influencing the shade matching performance of dentists and dental technicians when using two different shade guides. Br. Dent. J. 2011, 211, 23. [CrossRef] [PubMed]

54. Hardan, L. Protocols for Mobile Dental Photography with Auxiliary Lighting. J. Craniomandib. Sleep Pract. $2021,39,181$.

55. Tam, W.-K.; Lee, H.-J. Accurate Shade Image Matching by Using a Smartphone Camera. J. Prosthodont. Res. 2017, 61, 168-176. [CrossRef]

56. Sampaio, C.S.; Gurrea, J.; Gurrea, M.; Bruguera, A.; Atria, P.J.; Janal, M.; Bonfante, E.A.; Coelho, P.G.; Hirata, R. Dental Shade Guide Variability for Hues B, C, and D Using Cross-Polarized Photography. Int. J. Periodontics Restor. Dent. 2018, 38, s113-s118. [CrossRef] [PubMed]

57. Gurrea, J.; Gurrea, M.; Bruguera, A.; Sampaio, C.S.; Janal, M.; Bonfante, E.; Coelho, P.G.; Hirata, R. Evaluation of Dental Shade Guide Variability Using Cross-Polarized Photography. Int. J. Periodontics Restor. Dent. 2016, 36, e76-e81. [CrossRef] [PubMed]

58. Johnston, W.M.; Kao, E.C. Assessment of Appearance Match by Visual Observation and Clinical Colorimetry. J. Dent. Res. 1989, 68, 819-822. [CrossRef]

59. Wee, A.G.; Lindsey, D.T.; Kuo, S.; Johnston, W.M. Color Accuracy of Commercial Digital Cameras for Use in Dentistry. Dent. Mater. 2006, 22, 553-559. [CrossRef] 
60. Monterubbianesi, R.; Tosco, V.; Bellezze, T.; Giuliani, G.; Özcan, M.; Putignano, A.; Orsini, G. A Comparative Evaluation of Nanohydroxyapatite-Enriched Hydrogen Peroxide Home Bleaching System on Color, Hardness and Microstructure of Dental Enamel. Materials 2021, 14, 3072. [CrossRef]

61. Chu, S.J.; Trushkowsky, R.D.; Paravina, R.D. Dental color matching instruments and systems. Review of clinical and research aspects. J. Dent. 2010, 38, 2-16. [CrossRef] [PubMed]

62. Hardan, L.S.; Moussa, C. Mobile Dental Photography: A Simple Technique for Documentation and Communication. Quintessence Int. 2020, 51, 510-518. [PubMed]

63. Moussa, C.; Hardan, L.; Kassis, C.; Bourgi, R.; Devoto, W.; Jorquera, G.; Panda, S.; Abou Fadel, R.; Cuevas-Suárez, C.E.; LukomskaSzymanska, M. Accuracy of Dental Photography: Professional vs. Smartphone's Camera. BioMed Res. Int. 2021, $2021,3910291$. [CrossRef] [PubMed] 